\title{
Whole-genome sequencing identifies a homozygous deletion encompassing exons 17 to 23 of the integrin beta 4 gene in a Charolais calf with junctional epidermolysis bullosa
}

\author{
Pauline Michot ${ }^{1,2}$, Oscar Fantini ${ }^{3}$, Régis Braque ${ }^{4}$, Aurélie Allais-Bonnet ${ }^{2,5}$, Romain Saintilan ${ }^{1,2}$, Cécile Grohs ${ }^{1}$, \\ Johanna Barbieri', Lucie Genestout ${ }^{7}$, Coralie Danchin-Burge ${ }^{8}$, Jean-Marie Gourreau' ${ }^{9}$, Didier Boichard ${ }^{1}$, \\ Didier $\operatorname{Pin}^{3^{*}}$ and Aurélien Capitan ${ }^{1,2^{*}}$
}

\begin{abstract}
Background: Since 2010, four Charolais calves with a congenital mechanobullous skin disorder that were born in the same herd from consanguineous matings were reported to us. Clinical and histopathological examination revealed lesions that are compatible with junctional epidermolysis bullosa (JEB).

Results: Fifty-four extended regions of homozygosity (>1 Mb) were identified after analysing the whole-genome sequencing (WGS) data from the only case available for DNA sampling at the beginning of the study. Filtering of variants located in these regions for (i) homozygous polymorphisms observed in the WGS data from eight healthy Charolais animals and (ii) homozygous or heterozygous polymorphisms found in the genomes of 234 animals from different breeds did not reveal any deleterious candidate SNPs (single nucleotide polymorphisms) or small indels. Subsequent screening for structural variants in candidate genes located in the same regions identified a homozygous deletion that includes exons 17 to 23 of the integrin beta 4 (ITGB4), a gene that was previously associated with the same defect in humans. Genotyping of a second case and of six parents of affected calves (two sires and four dams) revealed a perfect association between this mutation and the assumed genotypes of the individuals. Mining of Illumina BovineSNP50 Beadchip genotyping data from 6870 Charolais cattle detected only 44 heterozygous animals for a 5.6-Mb haplotype around ITGB4 that was shared with the carriers of the mutation. Interestingly, none of the 16 animals genotyped for the deletion carried the mutation, which suggests a rather recent origin for the mutation.
\end{abstract}

Conclusions: In conclusion, we successfully identified the causative mutation for a very rare autosomal recessive mutation with only one case by exploiting the most recent DNA sequencing technologies.

\section{Findings}

Hereditary junctional epidermolysis bullosa (JEB) is a recessive inherited blistering disorder of the skin and mucous membrane in which tissue separation occurs within the lamina lucida (i.e. under the basal plasma membrane of the basal keratinocytes and above the basement membrane) of the basement membrane zone

\footnotetext{
* Correspondence: didier.pin@vetagro-sup.fr; aurelien.capitan@jouy.inra.fr ${ }^{3}$ Université de Lyon, VetAgro Sup, UPSP 2011-03-101 Interactions Cellules Environnement, 1 avenue Bourgelat, Marcy l'Etoile F-69280, France 'INRA, UMR1313 Génétique Animale et Biologie Intégrative, domaine de Vilvert, Jouy-en-Josas F-78352, France

Full list of author information is available at the end of the article
}

(BMZ) at the dermal-epidermal junction [1]. This rare mechanobullous disease was previously reported to be associated with mutations in genes encoding components of the hemidesmosome anchoring complex (ITGA6, ITGB4, COL17A1, and LAMA3, LAMB3, and LAMC2, encoding the subunit polypeptides of laminin 5) in humans and in several other mammalian species [2-8]. Congenital JEB has been sporadically observed over the past 30 years in Charolais cattle but, to date, the causative mutation has not been identified.

Since 2010, four cases (three males and one female) that were born in the same French herd from consanguineous 
matings were reported to us. Analysis of the pedigree data revealed that all JEB-affected animals trace back, on both the maternal and paternal sides, to a single founder bull born in Great Britain in 1986 (Figure 1), which suggests an autosomal recessive mode of inheritance as the most parsimonious model.

At birth, acral clinical lesions were observed with dysungulation of the four hooves and erosions and ulcers of the skin from the carpal and tarsal joints, fetlocks, ears, eyes, and muzzle and oral cavity (i.e. nares, tongue, buccal and labial sides of the mucosa and palate) (Figure 2). In addition, three of the four cases showed ear deformities (atrophied pinna and closed ears) and one displayed major epidermal loss on the back after being licked by its mother. General signs included anorexia, apathy, emaciation and marked cutaneous pain that justified rapid euthanasia.

Histopathological examination of two cases revealed sub-epithelial splitting and blistering without keratinocyte cytolysis (Figure 3). The basal keratinocytes appeared to be intact. Periodic acid Schiff (PAS) staining was weakly positive for the basement membrane that was located at the base of the blisters. Cleft formation was sometimes present around hair follicles. Dermal inflammatory infiltrate was of varying degrees, very mild in non-ulcerated areas but marked in ulcerated areas.
Because of the rare occurrence of JEB in Charolais cattle and of rapid euthanasia of affected animals, only one case (out of the three that were born and reported at the beginning of the study) was available for DNA sampling, thus preventing the use of a classical autozygosity mapping approach $[9,10]$. As a consequence, we decided to sequence the whole genome (WGS) of this animal and applied an alternative strategy as described in Lupski et al. [11]. DNA was extracted from the pinna using the DNeasy Blood and Tissue Kit (Qiagen). One paired-end library with a 450-bp insert size was generated with the NEXTflex PCR-Free DNA Sequencing Kit (Bioscientific) and sequenced on one lane of the HiSeq 2000 platform (Illumina) with Illumina TruSeq V3 Kit (200 cycles).This has been submitted to the NCBI Sequence Read Archive under the accession number SRP055078. The 101-bp reads were mapped on the UMD3.1 bovine sequence assembly using BWA [12]. Reads with multiple alignments were removed (yielding a final average sequence coverage of $11.4 \mathrm{X}$ ) and variants were called using SAMtools [13].

Then, the genome of this consanguineous animal was screened for extended regions of homozygosity. To avoid artifactual heterozygous genotypes in homozygous regions due to pseudo-SNPs or sequencing errors, only 706791 SNPs from the Illumina Bovine HD Beadchip were used. A total of 54 blocks with a minimal size of $1 \mathrm{Mb}$ and

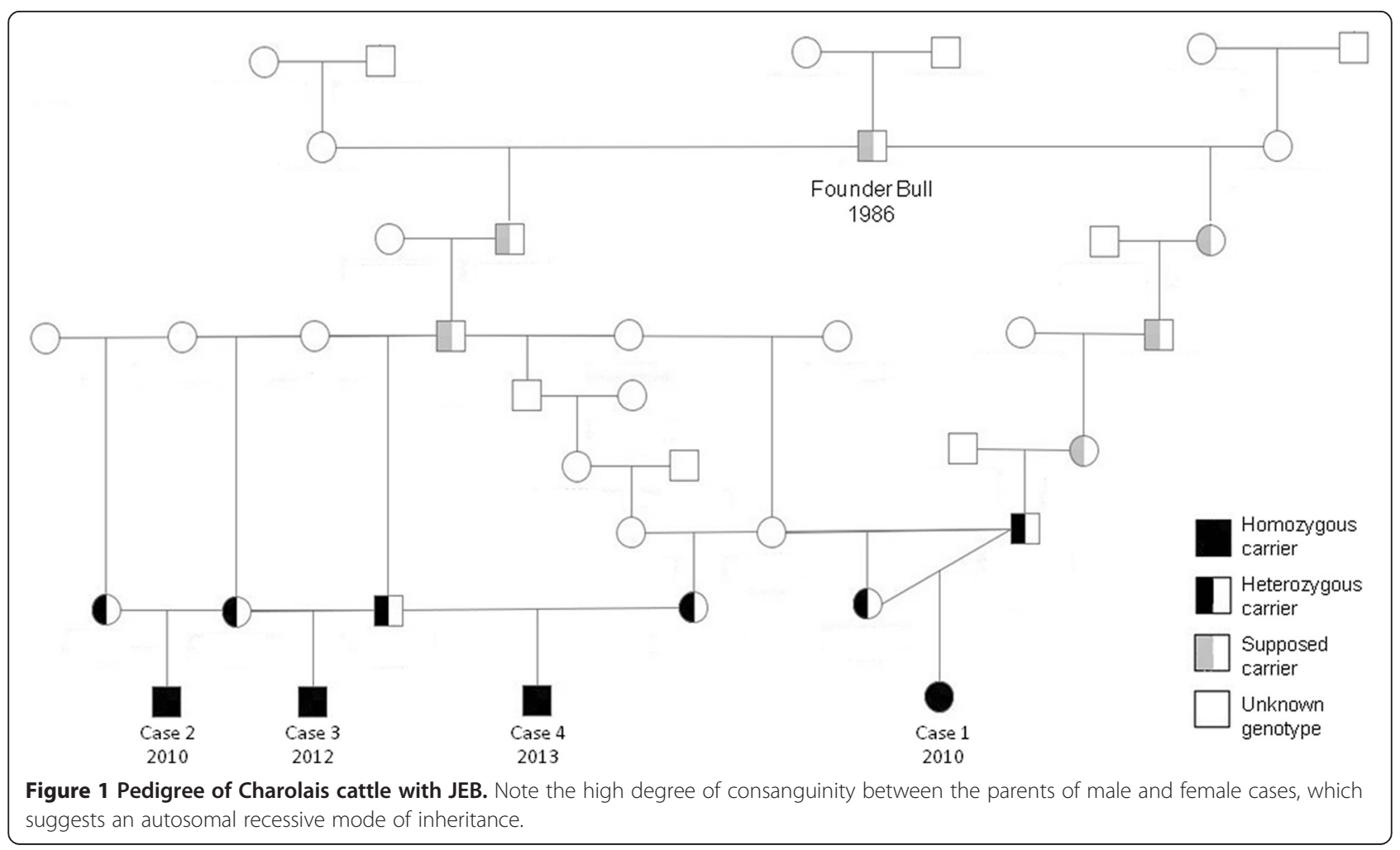




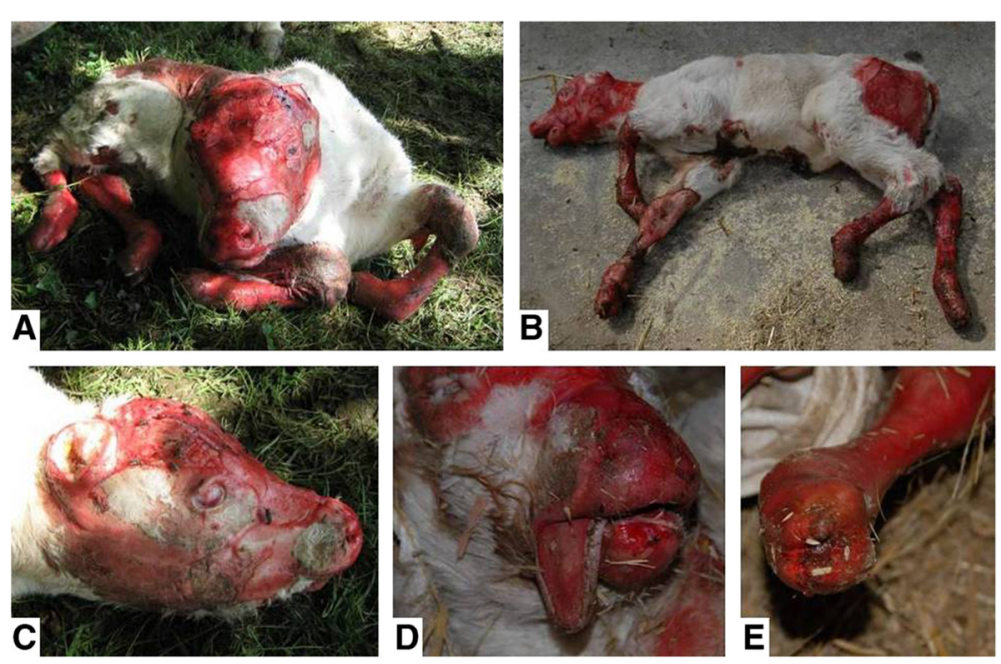

Figure 2 Clinical features of recessive JEB in Charolais cattle. (A) and (B) Global views of cases 3 and 2, respectively. (C) Head from case 3 showing atrophied pinna and skin lesions on the eyes, face and muzzle. (D) Lesions of the muzzle and tongue from case 1. (E) Forelimb from case 1 with dysungulation. These photos are personal photographs.

containing only homozygous genotypes were identified (Figure 4A).

SNPs and small Indels located in these blocks and with a quality score greater than 30 were annotated using Ensembl VEP [14] and filtered for (i) homozygous polymorphisms observed in the WGS data from eight healthy Charolais animals and (ii) homozygous or heterozygous polymorphisms found in the genomes of 234 animals from different breeds [15] (assuming that the causative mutation is recessive and specific to the Charolais cattle). No homozygous
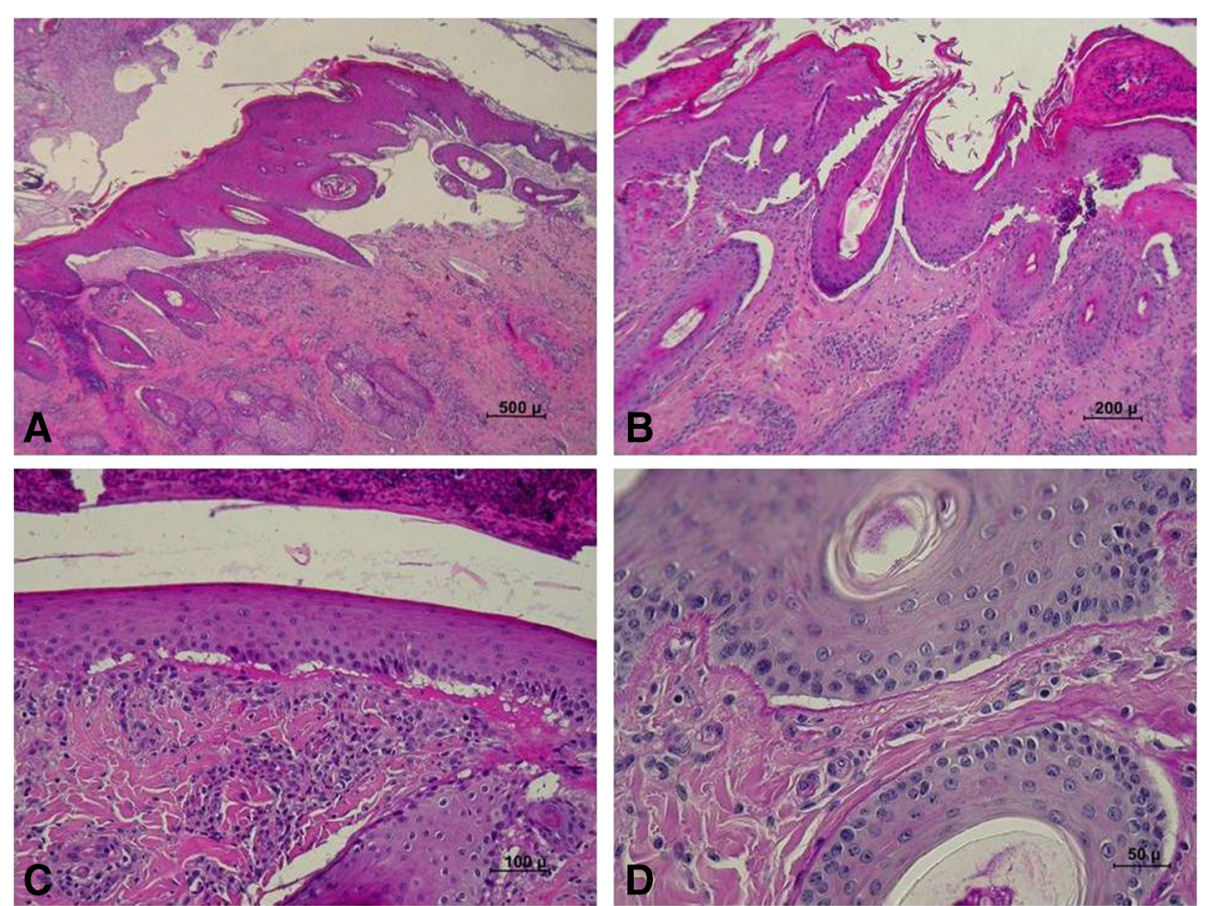

Figure 3 Histopathological features of JEB in Charolais cattle. (A) Large area of sub-epithelial splitting and blistering. (B) Sub-epithelial splitting and blistering, and cleft formation around hair follicles. (C) Vacuolation beneath basal keratinocytes of the epidermis and fibrin deposition on the dermal side of the vacuoles (5- $\mu \mathrm{m}$ section of tissue embedded in paraffin and stained with haematoxylin and eosin). (D) Vacuolation beneath basal keratinocytes of the epidermis and above the periodic acid Schiff (PAS)-positive basement membrane; 5 - $\mu \mathrm{m}$ section of tissue embedded in paraffin and stained with PAS. 


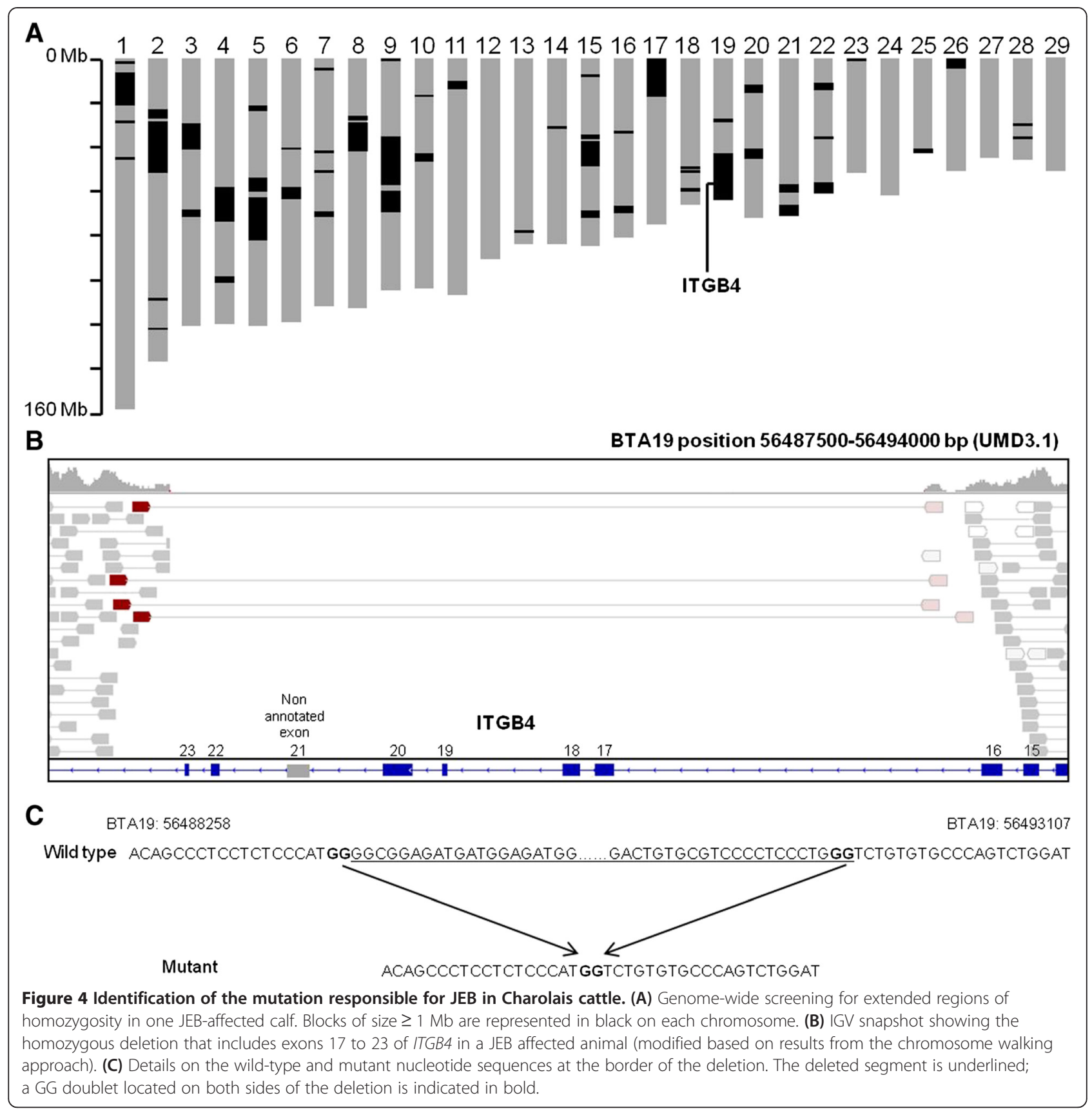

deleterious mutation (frame-shifts, in-frame insertions or deletions, stop gain or loss of variants as well as polymorphisms that affect splice donor or splice acceptor sites and missense polymorphisms predicted to be deleterious) was found with this approach.

In a second attempt, we investigated the content of the homozygous blocks and found only one gene that was previously reported to be involved in JEB: ITGB4, which encodes the integrin beta 4 protein. A subsequent screening for structural variants using the Integrative Genomics Viewer (IGV) [16] enabled us to identify a 4.8-kb deletion on bovine chromosome 19 or BTA19 (g.56488278_56493087del on the UMD3.1 assembly). Since there were two gaps, and at least one artifactual segmental duplication in the current bovine assembly within the region deleted in JEB, we built a local assembly to determine the exact nature of the mutation, which finally consists in a 2831-bp deletion encompassing exons 17 to 23 of the ITGB4 gene (Figure 4B, C and Additional file 1). This mutation was further confirmed by PCR-amplification of a 932-bp fragment that spans the deletion with the LEFT (TTCCCTGGGGGATCTGGGA) and RIGHT (CGTCTG 
CGAGATCAACTACT) primers using the Go-Taq Flexi DNA Polymerase (Promega) followed by Sanger sequencing (Eurofins MWG, Ebersberg Germany).

ITGB4 encodes the beta subunit of the alpha 6 beta 4 integrin heterodimer. This transmembrane receptor is a key component of the hemidesmosome anchoring complex which connects basal keratinocytes to the basement membrane by linking the extracellular N-termini of the $\alpha$ and $\beta$ subunits to laminin 5 whereas the intracellular C-termini are attached to the cytokeratin network via plectin or via the type XVII collagen and the bullous pemphigoid antigen 1 (BP230) [17-21] (Figure 5A). In humans, numerous mutations in ITGB4 have been reported to cause JEB with truncating mutations being associated with a more severe (and mostly lethal) phenotype [22]. In Charolais cattle, the deletion of exons 17 to 23 of ITGB4 is predicted to result in a complete deletion of the transmembrane domain of the protein and the joining of exon 16 to exon 24 causes a frameshift leading to the production of a protein in which all the intracellular domains in addition to the transmembrane domain (ITGB4 p.A665Gfs*11) are missing (Figure 5B). These extensive protein modifications are assumed to impair the association of ITGB4 with ITGA6 and other key proteins of the hemidesmosome anchoring complex. This is consistent with the absence of hybridization signals observed by Guaguere et al. [23] after immunohistochemical characterization of the skin of a JEB-affected Charolais calf with an antibody directed towards the alpha 6 beta 4 integrin complex.

Genotyping of a second case available for DNA sampling (born at the end of our study) and of the

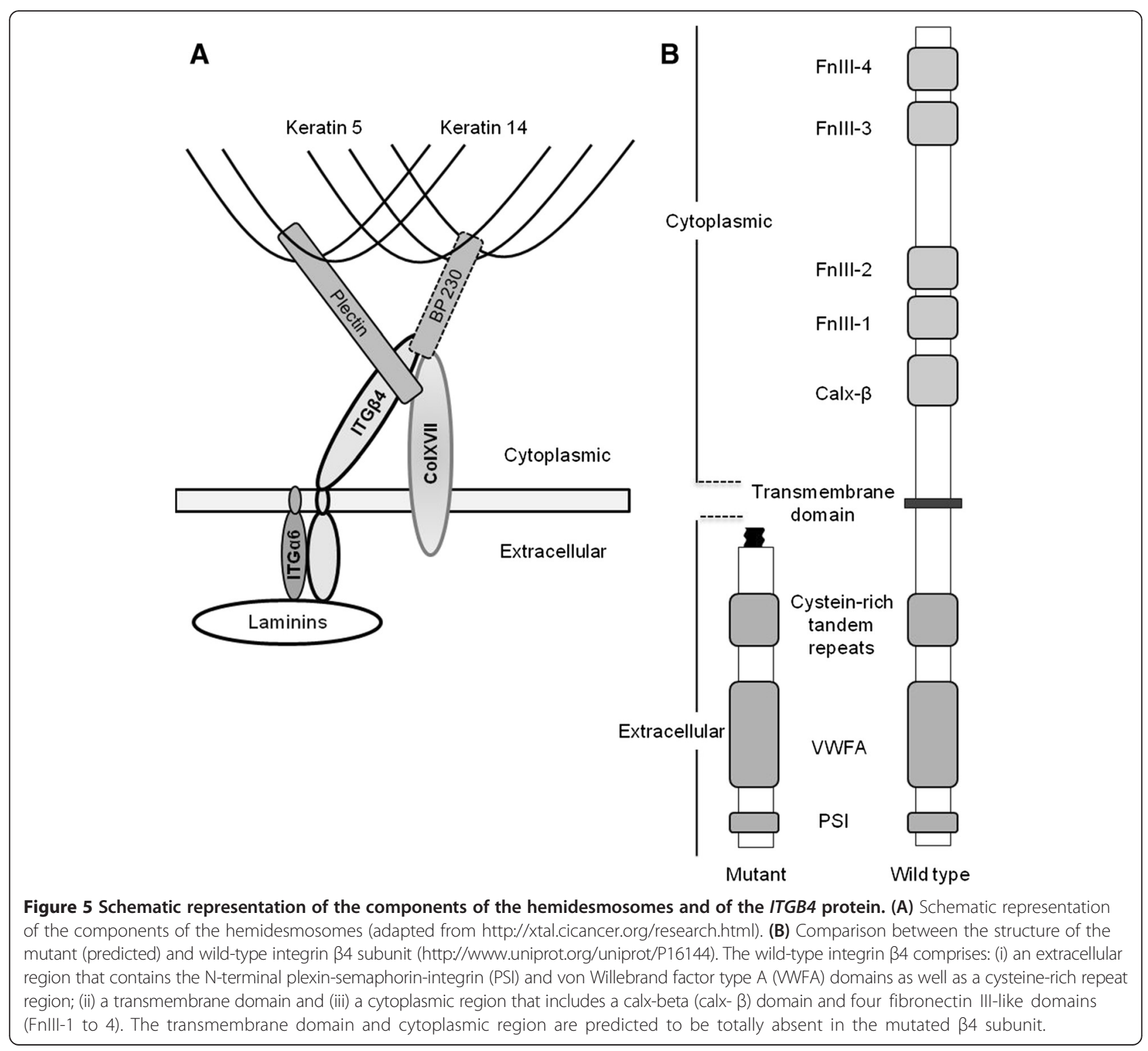



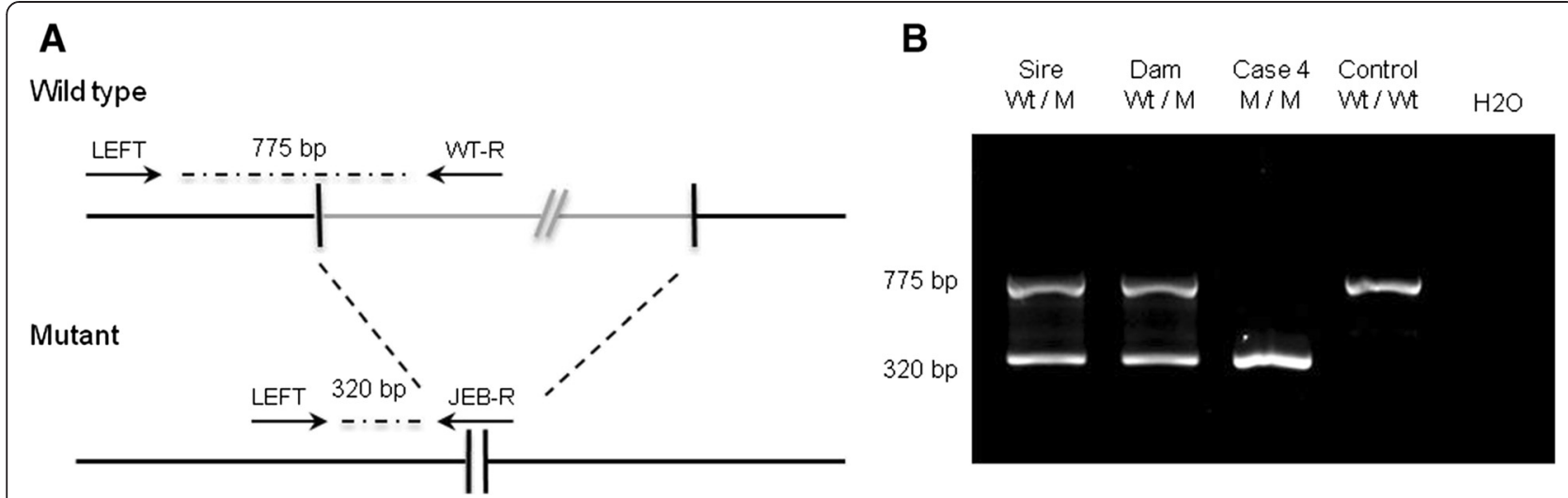

Figure 6 Genotyping of the deletion using a 3-primer PCR system. (A) Details on the design of the 3-primer PCR system. (B) Genotyping data of case 4 , its parents, and a homozygous wild-type animal (based on haplotype information).

six parents of the affected animals using a 3-primer PCR system (products amplified with primers LEFT, WT-R: TCTGCCCCACATGAATGCTT and JEB-R: AGACTGGG CACACAGACCAT using the same polymerase and revealed by electrophoresis on an ethidium-bromide-stained $2 \%$ agarose gel) revealed a perfect association between this mutation and the assumed genotypes of the individuals (Figure 6A and B).

Taken together, these arguments strongly support that this deletion within ITGB4 is responsible for autosomal recessive JEB in Charolais cattle.

In an attempt to estimate the frequency of this mutation in the Charolais population that is bred by artificial insemination (AI), four parents were genotyped with the Illumina BovineSNP50 Beachip and phased as described in Boichard et al. [24] together with 6870 Charolais animals previously genotyped with the same array for genomic selection. Analysis of these data identified 44 animals that were all heterozygous for a rare (frequency $=3.2 \%$ ) $5.6-\mathrm{Mb}$ segment (105 markers between positions 51796076 and $57397180 \mathrm{Mb}$ on BTA19) that was shared identical by descent (IBD) with the haplotype carrying the ITGB4 deletion. Surprisingly, subsequent genotyping of 16 of these animals with our 3-primer PCR system revealed that none were carriers of the deletion suggesting a rather recent origin for this mutation on the scale of the Charolais breed's history. While our analysis also suggests the absence of the ITGB4 deletion in the French AI population, it would be relevant to genotype the French natural mating population. Indeed the founder bull descends from animals exported to Great Britain at the end of the 1960's from this population and it has been used as bull sire in French breeding herds in the 1990's.

In conclusion, with only one case, we identified a mutation which appears to be necessary and sufficient to cause autosomal recessive junctional epidermolysis bullosa by exploiting the most recent DNA sequencing technologies. Targeted genotyping of at risk pedigrees with the genetic test that we developed will allow the rapid eradication of this rare genetic disease in Charolais cattle.

\section{Additional file}

Additional file 1: Building of a local sequence assembly for the region deleted in the JEB calf. This additional file provides details on the methodology used to build the local assembly of the deleted region in the JEB calf and the nucleotide sequence associated.

\section{Competing interests}

The authors declare that they have no competing interests.

\section{Authors' contributions}

$A C$ and DP conceived and coordinated the study. RB performed clinical examinations. DP and OF performed histological analyses. PM, $A C, A B, R S$, $C G, J B$ and $D B$ participated in the genetic study. RB, JMG, LG, CD and DB contributed material and data. AC, PM and DP wrote the manuscript and DB critically revised the manuscript. All authors read and approved the final manuscript.

\section{Acknowledgements}

The authors are grateful to the breeders, C Bodet and M Mayet, for excellent collaboration. We would also like to thank the Charolais Univers and Gènes Diffusion breeding companies for giving us access to DNA samples and genotyping data generated for genomic selection purposes and $Y$ Amigues for retrieving these DNA samples from the LABOGENA DNA bank. Finally, the help of $S$ Jurado (chambre d'Agriculture de la Nièvre) and G Fosserz (Herd Book Charolais) for reconstituting the pedigree of the affected family is highly appreciated. P Michot is recipient of a PhD grant from ALLICE and Apis Gène.

\section{Author details}

${ }^{1}$ INRA, UMR1313 Génétique Animale et Biologie Intégrative, domaine de Vilvert, Jouy-en-Josas F-78352, France. ${ }^{2}$ ALLICE, 149 rue de Bercy, Paris F-75012, France. ${ }^{3}$ Université de Lyon, VetAgro Sup, UPSP 2011-03-101 Interactions Cellules Environnement, 1 avenue Bourgelat, Marcy l'Etoile F-69280, France.

${ }^{4}$ Cabinet des Vignes de la Fontaine, 41 rue du faubourg de Moulins, Saint-Pierre le Moutier F-58240, France. ${ }^{5}$ UMR 1198 Biologie du Développement et Reproduction, domaine de Vilvert, Institut National de la Recherche Agronomique, Jouy-en-Josas F-78352, France. ${ }^{6}$ INRA, UMR1388 GenPhySE, GeT-PlaGe, Castanet-Tolosan F-31320, France. ${ }^{7}$ LABOGENA DNA, domaine de Vilvert, Jouy-en-Josas F-78352, France. ${ }^{8}$ Institut de l'Elevage, 149 rue de Bercy, Paris 12 F-75595, France. ${ }^{9}$ Unité de Pathologie du Bétail, Ecole Nationale Vétérinaire d'Alfort, 7 avenue du Général de Gaulle, Maisons-Alfort F-94704, France. 
Received: 5 November 2014 Accepted: 3 March 2015

Published online: 03 May 2015

\section{References}

1. Fine JD, Eady RA, Bauer EA, Bauer JW, Bruckner-Tuderman L, Heagerty A, et al. The classification of inherited epidermolysis bullosa (EB): report of the third international consensus meeting on diagnosis and classification of EB. J Am Acad Dermatol. 2008;58:931-50.

2. Pulkkinen L, Christiano AM, Airenne T, Haakana H, Tryggvason K, Uitto J. Mutations in the gamma-2 chain gene (LAMC2) of kalinin/laminin 5 in the junctional forms of epidermolysis bullosa. Nat Genet. 1994;6:293-8.

3. McGrath JA, Pulkkinen L, Christiano AM, Leigh IM, Eady RAJ, Uitto J. Altered laminin 5 expression due to mutations in the gene encoding the beta-3 chain (LAMB3) in generalized atrophic benign epidermolysis bullosa. J Invest Derm. 1995;104:467-74.

4. Vidal F, Aberdam D, Miquel C, Christiano AM, Pulkkinen L, Uitto J, et al. Integrin beta-4 mutations associated with junctional epidermolysis bullosa with pyloric atresia. Nat Genet. 1995;10:229-34.

5. McGrath JA, Gatalica B, Christiano AM, Li K, Owaribe K, McMillan JR, et al. Mutations in the 180-kD bullous pemphigoid antigen (BPAG2), a hemidesmosomal transmembrane collagen (COL17A1), in generalized atrophic benign epidermolysis bullosa. Nat Genet. 1995;11:83-6.

6. Vidal F, Baudoin C, Miquel C, Galliano M-F, Christiano AM, Uitto J, et al. Cloning of the laminin alpha-3 chain gene (LAMA3) and identification of a homozygous deletion in a patient with Herlitz junctional epidermolysis bullosa. Genomics. 1995:30:273-80.

7. Ruzzi L, Gagnoux-Palacios L, Pinola M, Belli S, Meneguzzi G, D'Alessio M, et al. Homozygous mutation in the integrin alpha- 6 gene in junctional epidermolysis bullosa with pyloric atresia. J Clin Invest. 1997;99:2826-31.

8. Bruckner-Tuderman L, McGrath JA, Clare Robinson E, Uitto J. Animal models of epidermolysis bullosa: update 2010. J Invest Derm. 2010;130:1485-8.

9. Lander ES, Botstein D. Homozygosity mapping: a way to map human recessive traits with the DNA of inbred children. Science. 1987;236:1567-70.

10. Charlier C, Coppieters W, Rollin F, Desmecht D, Agerholm JS, Cambisano N, et al. Highly effective SNP-based association mapping and management of recessive defects in livestock. Nat Genet. 2008:40:449-54.

11. Lupski JR, Reid JF, Gonzaga-Jauregui C, Rio Deiros D, Chen DCY, Nazareth $\mathrm{L}$, et al. Whole-genome sequencing in a patient with Charcot-Marie-Tooth neuropathy. N Engl J Med. 2010;362:1181-91.

12. Li H, Durbin R. Fast and accurate short read alignment with Burrows-Wheeler transform. Bioinformatics. 2009;25:1754-60.

13. Li H, Handsaker B, Wysoker A, Fennell T, Ruan J, Homer N, et al. 1000 genome project data processing subgroup: the sequence alignment/map format and SAMtools. Bioinformatics. 2009;25:2078-9.

14. McLaren W, Pritchard B, Rios D, Chen Y, Flicek P, Cunningham F. Deriving the consequences of genomic variants with the Ensembl API and SNP effect predictor. Bioinformatics. 2010;26:2069-70.

15. Daetwyler HD, Capitan A, Pausch H, Stothard P, van Binsbergen R, Brøndum RF, et al. Whole-genome sequencing of 234 bulls facilitates mapping of monogenic and complex traits in cattle. Nat Genet. 2014;46:858-65.

16. Robinson JT, Thorvaldsdóttir H, Winckler W, Guttman M, Lander ES, Getz G, et al. Integrative genomics viewer. Nat Biotechnol. 2011;29:24-6.

17. Hopkinson SB, Findlay K, DeHart GW, Jones JC. Interaction of BP180 (type XVII collagen) and alpha6 integrin is necessary for stabilization of hemidesmosome structure. J Invest Dermatol. 1998;111:1015-22.

18. Hopkinson SB, Jones JC. The $\mathrm{N}$ terminus of the transmembrane protein BP180 interacts with the N-terminal domain of BP230, thereby mediating keratin cytoskeleton anchorage to the cell surface at the site of the hemidesmosome. Mol Biol Cell. 2000;11:277-86.

19. Fontao L, Favre B, Riou S, Geerts D, Jaunin F, Saurat JH, et al. Interaction of the bullous pemphigoid antigen 1 (BP230) and desmoplakin with intermediate filaments is mediated by distinct sequences within their COOH terminus. Mol Biol Cell. 2003;14:1978-92.

20. Koster J, Geerts D, Favre B, Borradori L, Sonnenberg A. Analysis of the interactions between BP180, BP230, plectin and the integrin alpha6beta4 important for hemidesmosome assembly. J Cell Sci. 2003;116:387-99.

21. De Pereda JM, Lillo MP, Sonnenberg A. Structural basis of the interaction between integrin alpha6beta4 and plectin at the hemidesmosomes. EMBO J. 2009;28:1180-90.
22. Pulkkinen $L$, Rouan F, Bruckner-Tuderman L, Wallerstein R, Garzon M, Brown T, et al. Novel ITGB4 mutations in lethal and nonlethal variants of epidermolysis bullosa with pyloric atresia: missense versus nonsense. Am J Hum Genet. 1998;63:1376-87.

23. Guaguere E, Berg K, Degorce-Rubiales F, Spadafora A, Meneguzzi G. Junctional epidermolysis bullosa in a Charolais calf with deficient expression of integrin a6ß4. Vet Dermatol. 2004;15:28.

24. Boichard D, Guillaume F, Baur A, Croiseau P, Rossignol MN, Boscher MY, et al. Genomic selection in French dairy cattle. Anim Prod Sci. 2012;52:115-20.

\section{Submit your next manuscript to BioMed Central and take full advantage of:}

- Convenient online submission

- Thorough peer review

- No space constraints or color figure charges

- Immediate publication on acceptance

- Inclusion in PubMed, CAS, Scopus and Google Scholar

- Research which is freely available for redistribution 